\title{
Stable isotope labeling assisted liquid chromatography-tandem mass spectrometry for the analysis of perfluorinated carboxylic acids in serum samples
}

Shijuan Zhang ${ }^{\mathrm{a}, \mathrm{b},{ }^{*}, \text { Zhongyin Ji }}{ }^{\mathrm{c}}$, Zhiwei Sun ${ }^{\mathrm{a}, \mathrm{b}}$, Mingshuang Li ${ }^{\mathrm{a}}$, Cuncun Sheng ${ }^{\mathrm{a}}$, Mingbo Yue ${ }^{\mathrm{a}}$, Yanxin $\mathrm{Yu}^{\mathrm{a}}$, Guang Chen ${ }^{\mathrm{a}, \mathrm{b}}$, Jinmao You ${ }^{\mathrm{a}, \mathrm{b}, \mathrm{c}, *}$

${ }^{a}$ Shandong Province Key Laboratory of Life-Organic Analysis, Qufu Normal University, Qufu, PR China

${ }^{b}$ Key Laboratory of Pharmaceutical Intermediates and Analysis of Natural Medicine, Qufu Normal University, Qufu, PR China

${ }^{\mathrm{c}}$ Key Laboratory of Tibetan Medicine Research, Northwest Institute of Plateau Biology, Chinese Academy of Science, Xining, PR China

Correspondence: Shijuan Zhang and Jinmao You, Shandong Province Key Laboratory of Life-Organic Analysis, Qufu Normal University, Qufu, PR China

E-mail: sjzhang156@163.com(S. Zhang); jmyou6304@163.com (J. You)

Tel.:+86 5374456305

Fax: +86 5374456305 


\begin{abstract}
A new stable isotope labeling (SIL) reagent pair, 10-methyl-acridone-2-sulfonohydrazide (MASH) and its deuterated counterpart $\mathrm{d}_{3}-\mathrm{MASH}$ was synthesized and successfully applied to the analysis of perfluorinated carboxylic acids (PFCAs) in serum samples. The limits of detection (LODs) were in the range of $0.07-0.42 \mu \mathrm{g} / \mathrm{L}$, and the limits of quantitation (LOQs) were in the range of $0.25-1.38 \mu \mathrm{g} / \mathrm{L}$. Besides ionization enhancing effect, MASH also showed excellent fluorescence property. Therefore, the mass spectrometer operation cost was greatly lowered by carrying out parameter optimization experiments on HPLC which is easier to operate and maintain. The SIL strategy was confirmed to be effective in reducing matrix effect. The developed multiple-reaction monitoring (MRM) condition of PFCAs was also suitable for other carboxylic acid due to the introduction of MASH which is more prone to fragmentation than the analytes. With the MRM conditions obtained from PFCAs, fatty acids were also found in serum samples. This feature made the proposed method show powerful potential in the identification of acidic compounds in complex samples in the absence of corresponding standard.
\end{abstract}

Keywords: stable isotope labeling; PFCA; serum; fluorescence

\title{
1. Introduction
}

Perfluorinated compounds (PFCs) have attracted great concern because of their ubiquitous occurrence and potential carcinogenicity [1-3]. Due to their unique physicochemical characteristics, PFCs have been widely used in industrial and domestic fields. Perfluorinated carboxylic acids (PFCAs), especially perfluorooctanoic acid (PFOA), are important members of PFCs. They are resistant to typical environmental degradation processes and have been found in water, sediments, organisms, and even in 
human body [4].

Unlike other persistent organic pollutants which mainly accumulate in lipid, PFCAs bind to protein and accumulate in blood and viscus. Therefore, blood or serum samples were often used to evaluate human exposure to PFCAs. Matrix suppression and matrix enhancement effect was often observed in the analysis of samples with complex matrices [5-7].The accurate analysis of PFCAs in blood samples is also challenged by matrix effect. In many studies concerning PFCAs analysis, calibration standards had to be prepared in controlled matrix extract to compensate for matrix effect [8], but this means that more matrices were introduced into the instrument and more serious matrix effect might be observed in the long run.

Matrix effect can be reduced by using stable isotope analogues of the analytes as internal standards, but it is expensive and not practical to synthesize stable isotope analogues to all target compounds. Stable isotope labeling (SIL) strategy is one of the most important tools to overcome matrix effect. Two labeling reagents which have identical functional groups and differ only in isotopic composition are used for sample and standard derivatization respectively. The light and heavy versions of the labeling reagents show highly similar chemical property and thus the derivatization can be carried out under the same conditions. After derivatization, light and heavy labeled analytes are mixed and analyzed by liquid chromatography-tandem mass spectrometry (HPLC-MS/MS) [9-14]. The isotopic pairs of the labeled analytes are analyzed within a single run and thus have identical retention times. Therefore, matrix effects and ionization efficiencies of sample and standard are expected to be the same because they are electrosprayed from identical solution. 
SIL strategy is also characterized by its ionization-enhancing effect through the introduction of a permanently charged or easily protonated moiety into the analytes. Charge reversal derivatization can be achieved by this strategy to enhance the HPLC-MS/MS sensitivity of compounds like carboxylic acids and phenols [14,15]. Furthermore, derivatization increases the molecular weight of the analytes and makes them shift out of the low $\mathrm{m} / \mathrm{z}$ region which usually exhibits significant background noise either from solvent clusters or from the common contaminants which are often observed in LC-MS analysis[16].

In this work, a new SIL method was developed for the analysis of PFCAs in serum samples. Isotopic labeling reagent 10-methyl-acridone-2-sulfonohydrazide (MASH) and its deuterated counterpart $\mathrm{d}_{3}$-MASH were synthesized and applied to the SIL analysis of PFCAs. Unlike the previously reported SIL reagents which showed only ionization-enhancing property, MASH also showed excellent fluorescence property. Therefore, most of the parameter optimization work was carried out by HPLC with fluorescence detection. This was important for the maintenance of the mass spectrometer (MS) because the labeling reagent was often used in large excess in order to obtain sufficient labeling of the analytes. MS contamination was thus greatly reduced and the running cost was lowered correspondingly. The proposed method was successfully applied to the quantitation of PFCAs in serum samples. It also shows good potential in the multiple-reaction monitoring (MRM) of free fatty acids in the absence of standard.

\section{Experimental}

\subsection{Reagents and chemicals}

Perfluorohexanoic acid (C6), perfluoroheptanoic acid (C7), perfluorooctanoic acid 
(C8), perfluorononanoic acid (C9), perfluorodecanoic acid (C10) and perfluoroundecanoic acid (C11) were purchased from national standard material research center of China. Methanol, 1-ethyl-3-(3-dimethylaminopropyl)-carbodiimide hydrochloride (EDC), dichloromethane (DCA), N-Hydroxysuccinimide(NHS), n-hexane and acetonitrile were of HPLC grade and purchased from Sigma-Aldrich (USA). Water was purified on a Milli-Q system (Millipore, Bedford, MA, USA). All other reagents used were of HPLC grade or at least of analytical grade.

Individual stock solutions of $100 \mathrm{mg} / \mathrm{L}$ for all compounds were prepared in HPLC-grade acetonitrile and stored at $4{ }^{\circ} \mathrm{C}$ in the dark. Standard solutions containing all compounds were mixed and diluted with acetonitrile, and working solutions of all compounds and calibration concentrations were prepared by appropriate dilution of the stock solutions on the day of analysis.

\subsection{Synthesis of MASH and $d_{3}-M A S H$}

\subsubsection{Synthesis of 10-methyl-acridone-2-sulfonyl chloride(MASC) and its deuterated} counterpart $d_{3}-M A S C$

MASC and $\mathrm{d}_{3}$-MASC were synthesized in authors' laboratory according to the method described in our previous study [17]. Briefly, acridone reacted with iodomethane or $\mathrm{d}_{3}$-iodomethane to form 10-methyl-acridone. Then 10-methyl-acridone reacted with chlorosulfonic acid to synthesize MASC or $\mathrm{d}_{3}$-MASC.

\subsubsection{Synthesis of MASH and $d_{3}-M A S H$}

To a 100-mL flask, $5 \mathrm{~mL} 85 \%$ hydrazine hydrate and $5 \mathrm{~mL}$ dichloromethane were added. The mixed solution was stirred and cooled to $0{ }^{\circ} \mathrm{C}$. Then $20 \mathrm{~mL} 50 \mathrm{~g} / \mathrm{L}$ MASC dichloromethane solution was added dropwise within $10 \mathrm{~min}$. After stirring at $0{ }^{\circ} \mathrm{C}$ for $1 \mathrm{~h}$, 
the excess reagent was evaporated by a rotary vacuum evaporator. Then the residue was washed by water and ethanol respectively. The crude products were recrystallized from acetonitrile to afford a yellow crystal, yield $0.93 \mathrm{~g}(94 \%)$. The determined $\mathrm{m} / z[\mathrm{M}+\mathrm{H}]^{+}$ for MASH and $\mathrm{d}_{3}-\mathrm{MASH}$ were 304.0 and 307.0, respectively. The synthesis route was depicted in Fig.1.

\subsection{Sample extraction}

Serum samples which were obtained by centrifuging blood samples for $10 \mathrm{~min}$ at 4000 rpm were provided by Qufu People's Hospital (Qufu, China). All samples were stored at $4{ }^{\circ} \mathrm{C}$ before analysis. Proteins were precipitated by acetonitrile before usage to avoid its contamination of the instrument. This procedure did not lead to the loss of PFCAs which interacted with protein through electrostatic force and Van der Waals forces [18].The procedures were as follows: an aliquot of $100 \mu \mathrm{L}$ of serum was added into a $5 \mathrm{~mL}$ glass centrifuge tube. Then a $0.4 \mathrm{~mL}$ of acetonitrile was added. After vortexing for $1 \mathrm{~min}$, samples were centrifuged to remove the precipitation. The upper layer was evaporated to dryness before derivatization.

\subsection{Derivatizaiton of PFCAs}

Under the optimized conditions, $100 \mu \mathrm{L}$ acetonitrile, $100 \mu \mathrm{L} 2.0 \times 10^{-3} \mathrm{~mol} / \mathrm{L}$ MASH, $10 \mu \mathrm{L}(0.02 \mathrm{~mol} / \mathrm{L}) \mathrm{EDC}$ and $10 \mu \mathrm{L}(0.03 \mathrm{~mol} / \mathrm{L}) \mathrm{NHS}$ were added into a vial containing dried sample. The vial was sealed and allowed to react in a water bath at $70^{\circ} \mathrm{C}$ for $30 \mathrm{~min}$. Standard samples were derivatized under the same conditions with $\mathrm{d}_{3}$ - MASH as labeling reagent. The derivatization procedure is shown in Fig. 1. After the reaction was completed, the mixture was cooled to room temperature and diluted to $0.5 \mathrm{~mL}$ by acetonitrile. Samples and standards were mixed in equal volume and then syringe filtered 
using a $0.22 \mathrm{~mm}$ nylon filter. The mixed samples were injected for LC-MS/MS analysis. Each sample was analyzed in three replicates.

\subsection{HPLC-MS/MS analysis}

HPLC-MS/MS analysis was performed on an Agilent 1290 series HPLC system coupled with an Agilent 6460 Triple Quadrupole MS/MS mass spectrometer (Agilent, Santa Clara, USA) equipped with an Agilent Jet Stream electrospray ionization source (ESI source). HPLC separation was achieved using a SB C18 column $(2.1 \times 50 \mathrm{~mm}, 1.8$ $\mu \mathrm{m}$ i.d., Agilent). Eluent A was $0.1 \%$ formic acid in $5 \%$ acetonitrile and B was $0.1 \%$ formic acid in acetonitrile. The flow rate was $0.25 \mathrm{~mL} / \mathrm{min}$ and the column temperature was kept at $30{ }^{\circ} \mathrm{C}$. The elution conditions were as follows: $20-90 \%$ B from 0 to 5 min and then hold for $5 \mathrm{~min}$. The eluent of the first 3 min which was mainly composed of excess labeling reagent was converted to waste. The injection volume was $2 \mu \mathrm{L}$. The mass spectrometer was operated in a positive ion mode for the monitoring of $[\mathrm{M}+\mathrm{H}]^{+}$. The optimal ESI source conditions were: capillary voltage $+4.0 \mathrm{kV}$; nebulizer 40 psi; dry gas $11.0 \mathrm{~L} / \mathrm{min}$; dry temperature $300{ }^{\circ} \mathrm{C}$; Sheath gas temperature $280{ }^{\circ} \mathrm{C}$; Sheath gas flow $10 \mathrm{~L} / \mathrm{min}$. MRM parameter optimization was carried out in the sequence of precursor ions scanning, fragmentor optimization, production ions scanning, collision energy (CE) optimization and ESI source parameter optimization. Each time only one parameter was optimized, the parameter which gave out the highest signal was applied in later study. The MRM parameters of the target compounds were as follows: the precursor ions for light labeled C6, C7, C8, C9, C10 and C11 were $\mathrm{m} / \mathrm{z}, 600.0, \mathrm{~m} / \mathrm{z}, 650.0, \mathrm{~m} / \mathrm{z}, 700.0, \mathrm{~m} / \mathrm{z}$ $750.0, \mathrm{~m} / \mathrm{z} 800.0$ and $\mathrm{m} / \mathrm{z} 850.0$, respectively. The precursor ions for heavy labeled analytes were $3 \mathrm{~m} / \mathrm{z}$ higher than those of the corresponding light ones. The production 
ions for all light labeled analytes were $\mathrm{m} / \mathrm{z} 208.0, \mathrm{~m} / \mathrm{z} 271.8$ and $\mathrm{m} / \mathrm{z} 224.0$, while the production ions for all heavy labeled analytes were $\mathrm{m} / \mathrm{z} 211.0, \mathrm{~m} / \mathrm{z} 274.8$ and $\mathrm{m} / \mathrm{z} 227.0$. The fragmentor for all analytes was $160 \mathrm{~V}$, and the CE for all analytes was $30 \mathrm{~V}$. Derivatization parameter optimization was carried out on HPLC 1260 coupled with a fluorescence detector (model G1321B, Agilent) with wavelengths of 262 and $425 \mathrm{~nm}$ for excitation and emission, respectively. The HPLC separation was carried out on a Hypersil Gold column $(4.6 \times 250 \mathrm{~mm}, 5 . \mu \mathrm{m}$ i.d., Thermo, USA), and its gradient conditions were as follows: $50-100 \% \mathrm{~B}$ from 0 to $8 \mathrm{~min}$ and then hold for $2 \mathrm{~min}$.

\section{6 method validation}

Limits of detection (LODs) and limits of quantitation (LOQs) were calculated at a signal-to-noise $(\mathrm{S} / \mathrm{N})$ ratio of 3 and 10 respectively. Linearity was obtained by comparing the experimental concentration ratios of light/heavy labeled standard with the theoretical ratios. The six ratio levels were 1:20, 1:5, 1:1, 5:1, 10:1 and 20:1, respectively. Recoveries were carried out by spiking blank serum samples with $1.0,3.0$ and $5.0 \mu \mathrm{g} / \mathrm{L}$ of standard solutions. Intra-day precision was determined by analyzing blank serum samples spiked at the same three levels of standards with six replicates, and inter-day precision was determined by running blank serum samples with spiked standards at the same levels with three replicates on three different days over a period of one week. Matrix effect was evaluated by spiking serum samples with three concentrations of $1.5,3.0$ and $5.0 \mu \mathrm{g} / \mathrm{L}$ of PFCAs with three replicates and analyzed by direct LC-MS/MS and SIL LC-MS/MS. All samples were light labeled and the standard samples were heavy labeled. Samples and standard were mixed in equal volume before MS analysis. Concentration ratios were obtained by comparing the peak abundance of samples with those of the standard sample 
of $5 \mu \mathrm{g} / \mathrm{L}$. If the ratio was beyond the linearity range, sample or standard should be diluted correspondingly.

\section{Result and discussion}

\subsection{Optimization of derivatization conditions}

MASH shows ionization-enhancing effect through the formation of a stable quaternary ammonium ion through the intramolecular isomerization [19]. Unlike the often used MS labeling reagents which show neither fluorescence property nor UV absorption, MASH shows excellent fluorescence property with excitation and emission wavelength of 262 and $425 \mathrm{~nm}$, respectively (Fig. 2). This feature can effectively alleviate the MS contamination and lower the MS running cost. Parameter optimization can be carried out on HPLC with no contamination of the MS which is expensive to maintain and clean. In this study, derivatization parameter optimization was carried out on HPLC 1260 system with fluorescence detection. To ensure the sufficient labeling of the analytes, parameters such as MASH and EDC concentration, derivatizing temperature and time were studied in detail. Containing identical functional group, the reaction activity of MASH and $\mathrm{d}_{3}$-MASH can be considered equal. Therefore, the most of derivatization conditions were optimized with MASH as labeling reagent unless otherwise stated. A representative HPLC chromatogram of MASH labeled PFCAs was shown in Fig. 3.

\subsubsection{Effect of temperature and time on derivatization}

The influence of temperature on derivatization was studied in the temperature range

of room temperature to $100{ }^{\circ} \mathrm{C}$. The MASH concentration was kept at $1.0 \times 10^{-3} \mathrm{~mol} / \mathrm{L}$, and the concentrations of NHS and EDC were all kept at $0.02 \mathrm{~mol} / \mathrm{L}$ according to our 
previous study and the study of Chen et al [20,21]. As shown in Fig.4, peak area increased along with temperature till $70^{\circ} \mathrm{C}$. Further increasing the temperature to $90^{\circ} \mathrm{C}$, no obvious change in peak area was observed. When temperature was higher than $90{ }^{\circ} \mathrm{C}$, the peak area decreased slightly. Highest peak area was obtained at $70 \circ \mathrm{C}$ for $30 \mathrm{~min}$. Increasing the derivatization time from $30 \mathrm{~min}$ to $1 \mathrm{~h}$, the peak areas of the derivatives were stable. This indicated that the sufficient labeling of the analytes could be finished within $30 \mathrm{~min}$.

\subsubsection{Effect of MASH concentration on derivatization}

The quantitation strategy of SIL differs from those traditional labeling strategies. Therefore, the labeling reagent concentration should be designed carefully to guarantee stable heavy and light labeling efficiency. In this study, both light and heavy MASH concentrations were studied in detail. The temperature was fixed at $70{ }^{\circ} \mathrm{C}$, and the reaction time was kept for $30 \mathrm{~min}$. The NHS and EDC concentrations were the same as those described in section 3.1.1. Constant peak intensities of light and heavy labeled analytes were achieved when MASH was seven-fold molar reagent excess to total molar analytes. Further increasing the excess of reagent beyond this level had no obvious effect on the yields. For the convenience of operation, $2.0 \times 10^{-3} \mathrm{~mol} / \mathrm{L}$ MASH was applied for PFCAs labeling. This concentration was sufficient enough for the analysis of PFCAs since the contents of acidic compounds in serum samples were far below this level. Under this condition, stable light and heavy peak area ratios were also obtained. The excess labeling reagent eluted before 3 min on the reversed phase column and was converted to waste instead of to MS to avoid the possible contamination of the instrument.

\subsubsection{Effects of EDC and NHS concentrations on derivatization}


EDC is one of the most often used condensing agents due to its excellent condensation and dehydration property. It can react with acids to form a highly unstable activated acid intermediate which could react easily with amine groups [20]. Therefore, sufficient EDC concentration is the guarantee of the complete reaction of PFCAs with MASH. The molar ratios of EDC to PFCAs were then studied in the range of $0.5-10$ by HPLC with fluorescence detection. The other parameters were fixed at the optimized conditions obtained in previous section. As shown in Fig. 4, the fluorescence intensity increased along with EDC concentration until the molar ratios of EDC to PFCAs reached 4. Further increasing the molar ratios to 6 , no enhancement in fluorescence intensity was observed. Considering the total concentration of $\mathrm{PFCA}_{\mathrm{S}}$ and free fatty acid which can also react with MASH in most serum samples were lower than $100 \mu \mathrm{mol} / \mathrm{L}[22,23], 10$ $\mu \mathrm{L}(0.02 \mathrm{~mol} / \mathrm{L}) \mathrm{EDC}$ was used as condensing agents for $100 \mu \mathrm{L}$ serum sample. Under this condition, the molar ratio of EDC to acid were higher than 4. It should be pointed out that EDC is sensitive to moisture and should be prepared everyday just before analysis. NHS can enhance the activity of carbonyl group and is often used simultaneously with EDC. According to the study of Chen et al [20], the NHS concentration was optimized in the concentration range of $0.015 \mathrm{~mol} / \mathrm{L}$ to $0.04 \mathrm{~mol} / \mathrm{L}$. The highest sensitivity was obtained when NHS concentration was $0.03 \mathrm{~mol} / \mathrm{L}$.

\subsection{Stability of the MASH labeled PFCAs}

Anhydrous acetonitrile solution of MASH could be stored $4{ }^{\circ} \mathrm{C}$ for one month with no decomposition. The stabilities of MASH labeled PFCAs were also investigated. Standard solution of $10 \mu \mathrm{g} / \mathrm{L}$ and a serum sample were repeatedly analyzed by LC-MS/MS after being placed at $4{ }^{\circ} \mathrm{C}$ for $1,2,3,4,6,8,10,15$ and $20 \mathrm{~d}$, respectively. 
The obtained peak abundance was stable with relative standard deviations of less than $6.6 \%$

\subsection{General MRM achieved by MASH labeling}

Derivatization converted the negative ionization mode of PFCAs to positive ionization mode which was believed to be more sensitive [15,24]. Meanwhile, an interesting phenomenon appeared. After derivatization, the MRM parameters of all PFCAs were equal. In contrast, the MRM parameters of PFCAs with no derivatization were different [4]. As shown in Fig. S1 in supporting information, MASH is a compound with a structure that is more prone to fragmentation than analytes themselves. Therefore, fragment ions of the derivatized analytes were mainly derived from MASH. Besides, the fragmentor voltage which was used to pull the precursor ions through the capillary was also governed by MASH which possessed a large rigid structure. Thus, the fragmentors for different compounds were similar.

This specific feature made the proposed method show great potential in the analysis of other carboxylic acids by a general MRM method which was first proposed by Tie et al [25]. In general MRM method, specific derivatization made the MRM parameters become universal for all analytes. The general MRM parameters can be obtained by several samples and then applied to all samples. In this study, the general MRM conditions achieved by MASH labeling made the qualitative analysis of acidic compound become possible in the absence of standard.

\subsection{Matrix effect}

Matrix effect challenges the accuracy of LC-MS by means of ionization competition $[26,27]$. It was frequently observed in the MS analysis of samples with complex matrices. 
SIL is one of the most important tools to overcome matrix effect through the co-elution of the sample and standard. In this study, the light and heavy MASH labeled PFCAs had almost identical retention times (Fig. 5) under the optimized conditions. Therefore, they were in the same matrix and experienced the same ionization competition process. To demonstrate the effect of SIL strategy in overcoming matrix effect, serum samples were spiked at three concentrations of $1.5,3.0$ and $5.0 \mu \mathrm{g} / \mathrm{L}$ of PFCAs with three replicates and analyzed by direct LC-MS/MS and SIL LC-MS/MS. The results were listed in Table 1. It is obvious that matrix effect aggravate along with increased retention time in direct LC-MS/MS analysis. In SIL LC-MS/MS method, the peak abundance of different PFCAs was not enhanced because matrices still existed and competed for ionization. However, since sample and standard eluted simultaneously within a single run, the ionization efficiencies of sample and standard could be considered equal. Therefore, matrix effect did not influence the accuracy of SIL LC-MS/MS method, and the obtained results were in good accordance with the spiked value.

\subsection{Method validation}

Calibration curves were constructed by comparing theoretic peak area ratios of $\mathrm{d}_{0}-/ \mathrm{d}_{3}-\mathrm{MASH}$ derivatives with the experimental peak area ratios. As shown in Table 2, good linearities were obtained with correlation coefficients $\left(\mathrm{R}^{2}\right)$ of higher than 0.996. LODs were in the range of $0.07-0.42 \mu \mathrm{g} / \mathrm{L}$, while LOQs were in the range of $0.25-1.38$ $\mu \mathrm{g} / \mathrm{L}$. Recoveries were carried out by spiking serum samples with $1.0,3.0$ and $5.0 \mu \mathrm{g} / \mathrm{L}$ of standard solutions. Intra-day precision was determined by analyzing samples spiked at the same three levels of standards with six replicates, and inter-day precision was determined by running samples with spiked standards at the same levels with three 
replicates on three different days over a period of one week. Satisfactory recoveries were obtained for all tested compounds (96.1-100.2\%). The intraday precision for the tested samples was in the range of $2.8-4.2 \%$, whereas the interday precision was between $4.0-6.3 \%$.

\subsection{Application}

The proposed method was applied to the analysis of PFCAs in serum samples. Serum samples were light labeled by $\mathrm{d}_{0}$-MASC and the standards were heavy labeled by $\mathrm{d}_{3}$-MASC. Samples and standards were mixed and analyzed by LC-MS/MS. In the analyzed 3 samples, C6 was found in two samples with concentrations of 0.86 and 3.20 $\mu \mathrm{g} / \mathrm{L}$. C8 was found in all the three samples and their concentrations were $3.53,6.28$ and $2.65 \mu \mathrm{g} / \mathrm{L}$, respectively. A representative chromatogram was shown in Fig. S2 in Supplementary Data.

The established general MRM method can also be well applied to the qualitative analysis of other compounds with carboxyl group. In this study, it was applied to the

analysis of free fatty acids in serum samples with no need of parent ion scanning, fragmentor optimization and CE optimization. The MRM parameters such as fragmentor, CE and production ions of PFCAs were used unchanged for fatty acids, while parent ions were calculated by molecular weight. As shown in Fig. 6, free fatty acid such as hexadecanoic acid, octadecanoic acid, tetradecanoic acid and linoleic acid were detected in serum samples by the developed method. Due to the complexity of serum samples, interference peaks were observed in some extracted ion chromatograms, but they could be excluded by retention time or isotope-induced retention time shift which was reported by Tie et al [28]. In this study, corresponding fatty acid standards were then heavy 
labeled and mixed with light labeled sample, the retention time of the detected fatty acid in serum were in good accordance with those of the heavy labeled standard (Fig. 6). Therefore, it can be concluded that the developed MRM method is general and can be directly applied to the analysis of new analytes.

\section{Conclusions}

In this study, a new SIL reagent pair was synthesized and successfully applied to the analysis of PFCAs in serum samples. Matrix effect was effectively reduced by the SIL strategy. Moreover, the SIL reagent also showed excellent fluorescence property. Therefore, parameter optimization was carried out by HPLC which greatly reduced the contamination of the MS instrument. Furthermore, the general MRM condition established by MASH labeling can be well applied to other compounds with carboxyl group in the absence of standard sample. The proposed method also shows powerful potential in the accurate analysis of acidic compounds in other samples with complex matrices.

\section{Acknowledgments}

The work was supported by Natural Science Foundation of Shandong Province (ZR2014BQ009), National Science Foundation of China (No. 21475075) and experimental research project of Qufu normal university (SJ201504).

\section{Reference}

[1] B. Jurado-Sanchez, E. Ballesteros, M. Gallego, Analytical method for biomonitoring of perfluoroalkyl acids in human urine, Talanta 128 (2014) 141-146.

[2] L. Yang, F. Jin, P. Zhang, Y. Zhang, J. Wang, H. Shao, M. Jin, S. Wang, L. Zheng, J. Wang, Simultaneous Determination of Perfluorinated Compounds in Edible Oil by Gel-Permeation Chromatography Combined with Dispersive Solid-Phase Extraction and Liquid Chromatography-Tandem Mass Spectrometry, J. Agric. Food Chem. 63 (2015) 8364-8371.

[3] S. Salihovic, A. Karrman, G. Lindstrom, P.M. Lind, L. Lind, B. van Bavel, A rapid method for the determination of perfluoroalkyl substances including structural isomers of perfluorooctane sulfonic acid in human serum using 96-well plates and column-switching ultra-high performance liquid chromatography tandem mass spectrometry, J. Chromatogr. A 1305 (2013) 164-170. 
[4] M. Lashgari, H.K. Lee, Determination of perfluorinated carboxylic acids in fish fillet by micro-solid phase extraction, followed by liquid chromatography-triple quadrupole mass spectrometry, J. Chromatogr. A 1369 (2014) 26-32.

[5] N. Guo, P. Liu, J. Ding, S.J. Zheng, B.F. Yuan, Y.Q. Feng, Stable isotope labeling - Liquid chromatography/mass spectrometry for quantitative analysis of androgenic and progestagenic steroids, Anal. Chim. Acta 905 (2016) 106-114.

[6] P.C. Mathias, J.A. Hayden, T.J. Laha, A.N. Hoofnagle, Evaluation of matrix effects using a spike recovery approach in a dilute-and-inject liquid chromatography-tandem mass spectrometry opioid monitoring assay, Clin. Chim. Acta 437 (2014) 38-42.

[7] A. Furey, M. Moriarty, V. Bane, B. Kinsella, M. Lehane, Ion suppression; a critical review on causes, evaluation, prevention and applications, Talanta 115 (2013) 104-122.

[8] C.R. Powley, S.W. George, T.W. Ryan, R.C. Buck, Matrix Effect-Free Analytical Methods for Determination of Perfluorinated Carboxylic Acids in Environmental Matrixes, Analy. Chem. 77 6353-6358.

[9] Y.H. Hao, Z. Zhang, L. Wang, C. Liu, A.W. Lei, B.F. Yuan, Y.Q. Feng, Stable isotope labeling assisted liquid chromatography-electrospray tandem mass spectrometry for quantitative analysis of endogenous gibberellins, Talanta 144 (2015) 341-348.

[10] M. Fernandez-Fernandez, P. Rodriguez-Gonzalez, M.E. Anon Alvarez, F. Rodriguez, F.V. Menendez, J.I. Garcia Alonso, Simultaneous determination of creatinine and creatine in human serum by double-spike isotope dilution liquid chromatography-tandem mass spectrometry (LC-MS/MS) and gas chromatography-mass spectrometry (GC-MS), Anal. Chem. 87 (2015) 3755-3763.

[11] M.G. Leeming, A.P. Isaac, B.J. Pope, N. Cranswick, C.E. Wright, J. Ziogas, R.A. O'Hair, W.A. Donald, High-resolution twin-ion metabolite extraction (HiTIME) mass spectrometry: nontargeted detection of unknown drug metabolites by isotope labeling, liquid chromatography mass spectrometry, and automated high-performance computing, Anal. Chem. 87 (2015) 4104-4109.

[12] Z. Sun, X. Wang, Y. Cai, J. Fu, J. You, Development of a pair of differential H/D isotope-coded derivatization reagents $\mathrm{d}(0) / \mathrm{d}(3)-4-(1-$ methyl-1H-phenanthro[9,10-d]imidazol-2-yl)phenlamine and its application for determination of aldehydes in selected aquatic products by liquid chromatography-tandem mass spectrometry, Talanta 120 (2014) 84-93.

[13] C. Song, S. Zhang, Z. Ji, Y. Li, J. You, Accurate Determination of Amino Acids in Serum Samples by Liquid Chromatography-Tandem Mass Spectrometry Using a Stable Isotope Labeling Strategy, J. Chromatogr. Sci. 53 (2015) 1536-1541.

[14] S. Zhang, Q. Yu, C. Sheng, J. You, Gas Purge Microextraction Coupled with Stable Isotope Labeling-Liquid Chromatography/Mass Spectrometry for the Analysis of Bromophenols in Aquatic Products, J. Agric. Food Chem. 64 (2016) 9452-9458.

[15] Y.Q. Huang, J.Q. Liu, H. Gong, J. Yang, Y. Li, Y.Q. Feng, Use of isotope mass probes for metabolic analysis of the jasmonate biosynthetic pathway, Analyst 136 (2011) 1515-1522.

[16] G. K., L. L., Differential ${ }^{12} \mathrm{C}-\mathrm{I}^{13} \mathrm{C}$-Isotope Dansylation Labeling and Fast Liquid Chromatography/Mass Spectrometry for Absolute and Relative Quantification of the Metabolome, Anal. Chem. 81 (2009) 3919-3932.

[17] S. Zhang, J. You, S. Ning, C. Song, Y.R. Suo, Analysis of estrogenic compounds in environmental and biological samples by liquid chromatography-tandem mass spectrometry with stable isotope-coded ionization-enhancing reagent, J. Chromatogr. A 1280 (2013) 84-91.

[18] T.-y. Hu, Q. Fang, Y. Jin, S.-s. Zhou, Y. Liu, A New Strategy to Probe and Compare the Binding Modes of Two Perfluorocarboxylic Acids with Human Serum Albumin Based on Spectroscopic and Molecular Docking Methods, Spectrosc. Spect. Anal. 36 (2016) 2698-2704.

[19] J. You, H. Zhao, Z. Sun, Y. Suo, G. Chen, 10-Ethyl-acridine-2-sulfonyl Chloride: A New Derivatization Agent for Enhancement of Atmospheric Pressure Chemical Ionization of Estrogens in Urine, Chromatographia 70 (2009) 45-55.

[20] S. Zhang, C. Song, G. Chen, L. Xia, X. Wang, J. You, A sensitive high-performance liquid chromatography method with fluorescence detection for the determination of fatty acids as exemplified forDendrobiumspecies, Eur. J. Lipid Sci. Tech. (2013) 1155-1163.

[21] J. Chen, Q. Lyu, M. Yang, Z. Chen, J. He, Selective elimination of the free fatty acid fraction from esterified fatty acids in rat plasma through chemical derivatization and immobilization on amino functionalized silica nano-particles, J. Chromatogr. A 1431 (2016) 197-204. 
[22] M. Lashgari, H.K. Lee, Micro-solid phase extraction of perfluorinated carboxylic acids from human plasma, J. Chromatogr. A 1432 (2016) 7-16.

[23] G.L. Li, G. Chen, Y.Q. Liu, N.H. Jing, J.M. You, A sensitive and selective HPLC-FLD method with fluorescent labeling for simultaneous detection of bile acid and free fatty acid in human serum, J. Chromatogr. B 895-896 (2012) 191-195.

[24] Y. Iwasaki, Y. Nakano, K. Mochizuki, M. Nomoto, Y. Takahashi, R. Ito, K. Saito, H. Nakazawa, A new strategy for ionization enhancement by derivatization for mass spectrometry, J. Chromatogr. B 879 (2011) 1159-1165.

[25] T. Cai, H. Ting, Z. Xin-Xiang, Z. Jiang, Z. Jin-Lan, HPLC-MRM relative quantification analysis of fatty acids based on a novel derivatization strategy, Analyst 139 (2014) 6154-6159.

[26] I. Marchi, V. Viette, F. Badoud, M. Fathi, M. Saugy, S. Rudaz, J.L. Veuthey, Characterization and classification of matrix effects in biological samples analyses, J. Chromatogr. A 1217 (2010) 4071-4078.

[27] W.C. Yang, J. Adamec, F.E. Regnier, Enhancement of the LC/MS Analysis of Fatty Acids through Derivatization and Stable Isotope Coding, Anal. Chem 79 (2007) 5150-5157.

[28] C. Tie, T. Hu, Z. X. Jia, J. L. Zhang, Automatic identification approach for high-performance liquid chromatography-multiple reaction monitoring fatty acid global profiling, Anal. Chem. 87 (2015) 8181-8185. 


\section{Figure captions}

Fig. 1. Schemes of synthesis and derivatization. (A) Synthesis routes of MASH and d $\mathrm{d}_{3}-\mathrm{MASH}$. (B) Reaction scheme of MASH with PFCAs. X = H or D.

Fig. 2. Fluorescence spectra of MASH. (A) Excitation spectra. (B) Emission structure.

Fig. 3. HPLC fluorescence chromatogram of MASH-PFCA derivative.

Fig. 4. Effects of EDC concentrations (A) and derivatization temperature (B) on derivatization.

Fig. 5. Extracted ion chromatograms of MASH (the lower chromatogram) and $\mathrm{d}_{3}-\mathrm{MASH}$ (the upper chromatogram) labeled PFCA standards with concentrations of $10 \mu \mathrm{g} / \mathrm{L}$ and $50 \mu \mathrm{g} / \mathrm{L}$ respectively.

Fig. 6. MRM chromatogram of fatty acid derivative. (A) Light labeled serum sample. (B) Heavy labeled standard. 


\section{Table 1}

Comparison of the accuracy of direct LC-MS/MS and SIL LC-MS/MS methods in serum sample analysis $(\mathrm{n}=3)$

\begin{tabular}{|c|c|c|c|c|c|}
\hline \multirow[t]{2}{*}{ Analyte } & \multirow{2}{*}{$\begin{array}{l}\text { Add amount } \\
\qquad(\mu \mathrm{g} / \mathrm{L})\end{array}$} & \multicolumn{2}{|l|}{ LC-MS/MS } & \multicolumn{2}{|c|}{ Stable isotope labeling LC-MS/MS } \\
\hline & & Determined amount $(\mu \mathrm{g} / \mathrm{L})$ & $\operatorname{RSD}(\%)$ & Determined amount $(\mu \mathrm{g} / \mathrm{L})$ & $\operatorname{RSD}(\%)$ \\
\hline \multirow[t]{3}{*}{ C6 } & 1.5 & 1.4 & 5.2 & 1.4 & 3.6 \\
\hline & 3.0 & 2.9 & 4.5 & 3.0 & 3.4 \\
\hline & 5.0 & 4.8 & 4.6 & 4.9 & 3.8 \\
\hline \multirow[t]{3}{*}{$\mathrm{C} 7$} & 1.5 & 1.4 & 4.9 & 1.5 & 3.5 \\
\hline & 3.0 & 2.8 & 5.2 & 2.9 & 3.2 \\
\hline & 5.0 & 4.8 & 4.7 & 4.9 & 3.0 \\
\hline \multirow[t]{3}{*}{$\mathrm{C} 8$} & 1.5 & 1.4 & 3.8 & 1.5 & 2.8 \\
\hline & 3.0 & 2.9 & 4.3 & 3.0 & 4.2 \\
\hline & 5.0 & 4.8 & 4.5 & 5.0 & 3.2 \\
\hline \multirow[t]{3}{*}{ C9 } & 1.5 & 1.4 & 5.8 & 1.5 & 4.1 \\
\hline & 3.0 & 2.6 & 5.3 & 3.0 & 3.8 \\
\hline & 5.0 & 4.7 & 4.9 & 5.0 & 3.5 \\
\hline \multirow[t]{3}{*}{$\mathrm{C} 10$} & 1.5 & 1.3 & 6.3 & 1.5 & 3.8 \\
\hline & 3.0 & 2.1 & 6.1 & 2.9 & 3.5 \\
\hline & 5.0 & 3.5 & 5.6 & 4.9 & 3.4 \\
\hline \multirow[t]{3}{*}{ C11 } & 1.5 & 1.0 & 7.9 & 1.5 & 4.2 \\
\hline & 3.0 & 1.9 & 7.6 & 3.0 & 3.6 \\
\hline & 5.0 & 3.9 & 6.8 & 4.9 & 3.5 \\
\hline
\end{tabular}




\section{Table 2}

Dynamical ratio range, linearity, LODs and LOQs of the SIL HPLC-MS/MS method.

\begin{tabular}{ccccccc}
\hline Analyte & $\begin{array}{c}\text { Dynamical ratio } \\
\text { range }\end{array}$ & Linearity & $\mathrm{R}^{2}$ & $\mathrm{LOD}(\mu \mathrm{g} / \mathrm{L})$ & $\mathrm{LOQ}(\mu \mathrm{g} / \mathrm{L})$ & $\begin{array}{c}\text { RSD of light/heavy } \\
\text { peak area ratio }(\%)\end{array}$ \\
\hline C6 & $20: 1-1: 20$ & $\mathrm{Y}=0.936 \mathrm{X}-0.026^{\mathrm{a}}$ & 0.996 & 0.07 & 0.25 & 2.54 \\
$\mathrm{C} 7$ & $20: 1-1: 20$ & $\mathrm{Y}=1.018 \mathrm{X}+0.054$ & 0.996 & 0.09 & 0.32 & 1.86 \\
$\mathrm{C} 8$ & $20: 1-1: 20$ & $\mathrm{Y}=0.999 \mathrm{X}-0.012$ & 0.996 & 0.12 & 0.45 & 1.69 \\
$\mathrm{C} 9$ & $20: 1-1: 20$ & $\mathrm{Y}=0.953 \mathrm{X}-0.037$ & 0.998 & 0.17 & 0.56 & 2.58 \\
$\mathrm{C} 10$ & $20: 1-1: 20$ & $\mathrm{Y}=0.972 \mathrm{X}$ & 0.999 & 0.20 & 0.62 & 2.50 \\
$\mathrm{C} 11$ & $20: 1-1: 20$ & $\mathrm{Y}=0.991 \mathrm{X}-0.031$ & 0.999 & 0.42 & 1.38 & 2.20 \\
\hline
\end{tabular}

${ }^{\mathrm{a}} \mathrm{X}$, theoretic concentration ratio of light/heavy labeled PFCAs; Y, experimental mass spectrometric peak intensity ratio of light/heavy labeled PFCAs. 

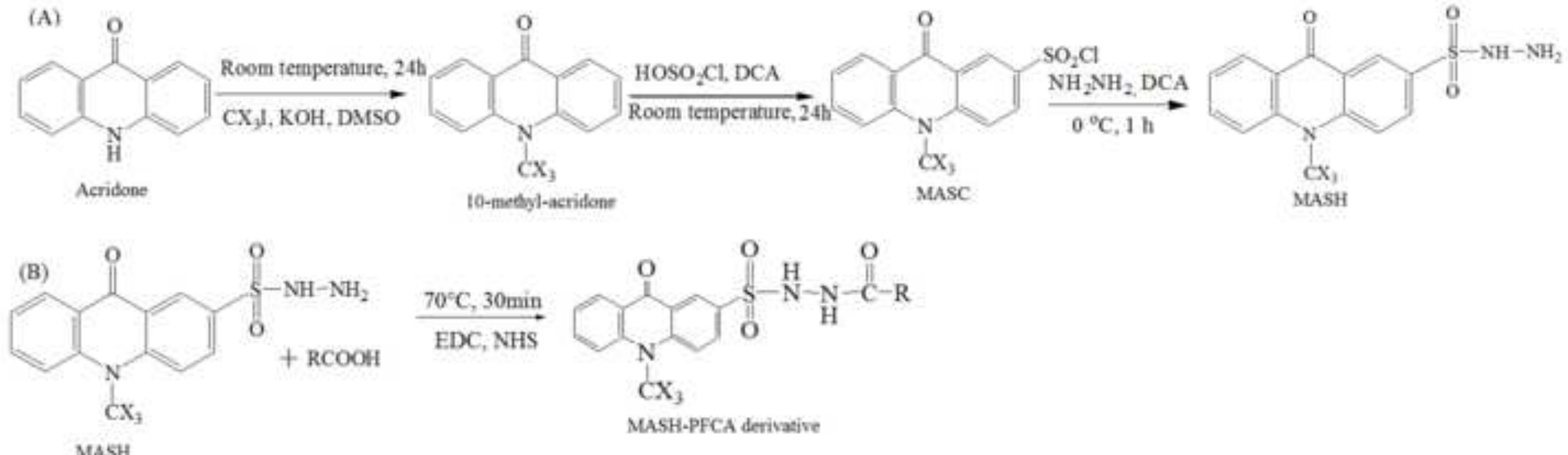

MASH 

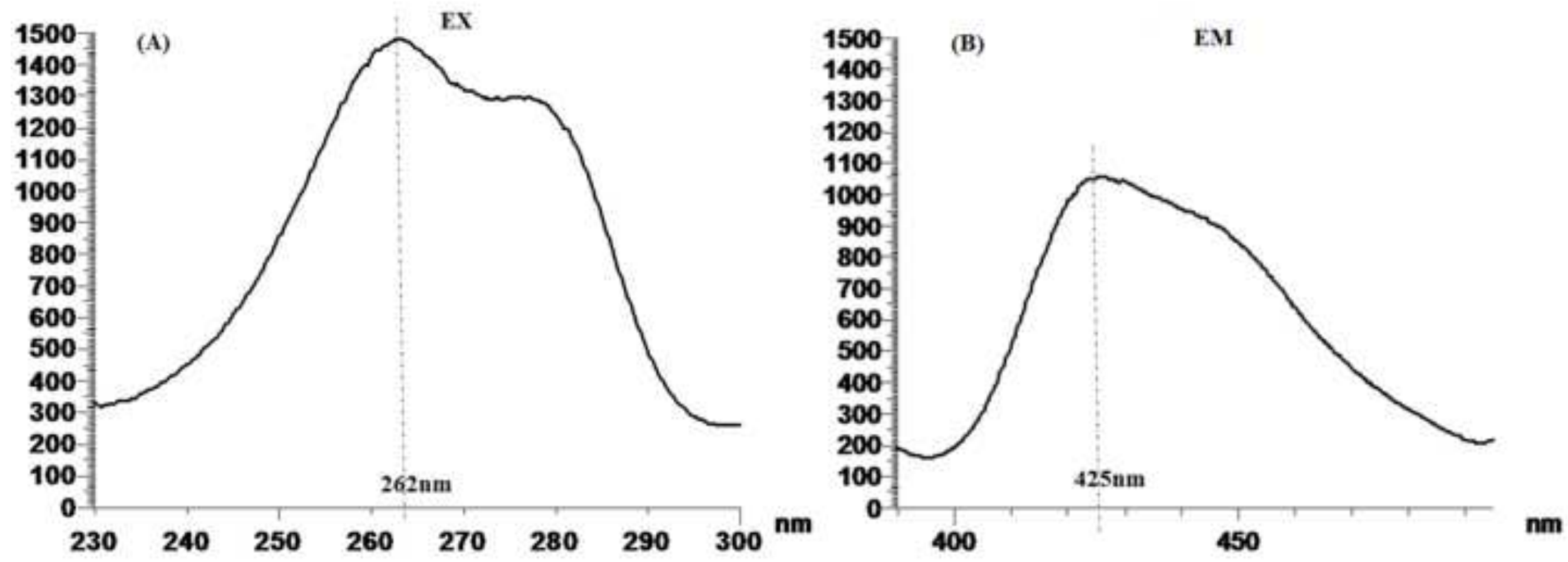


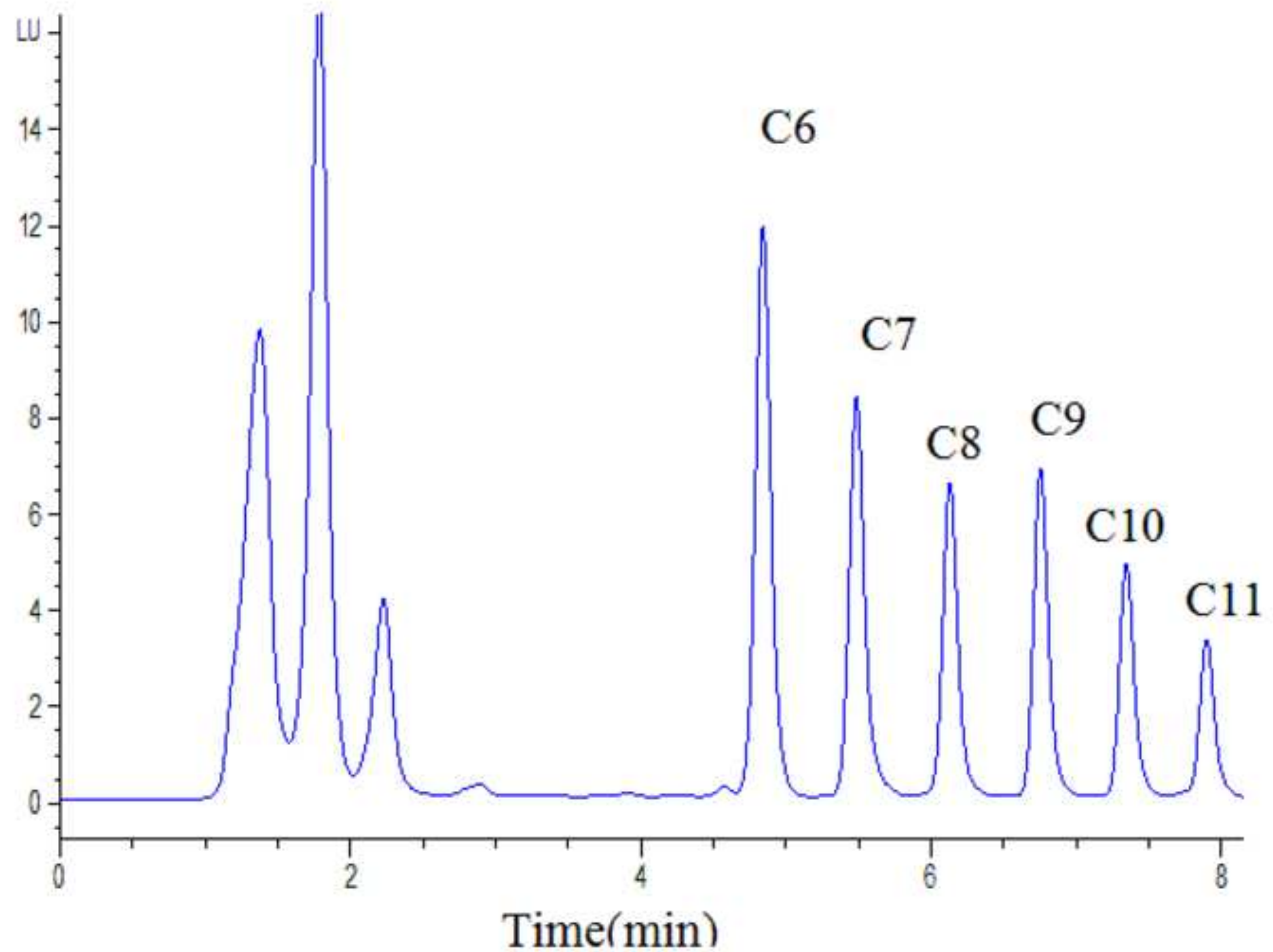



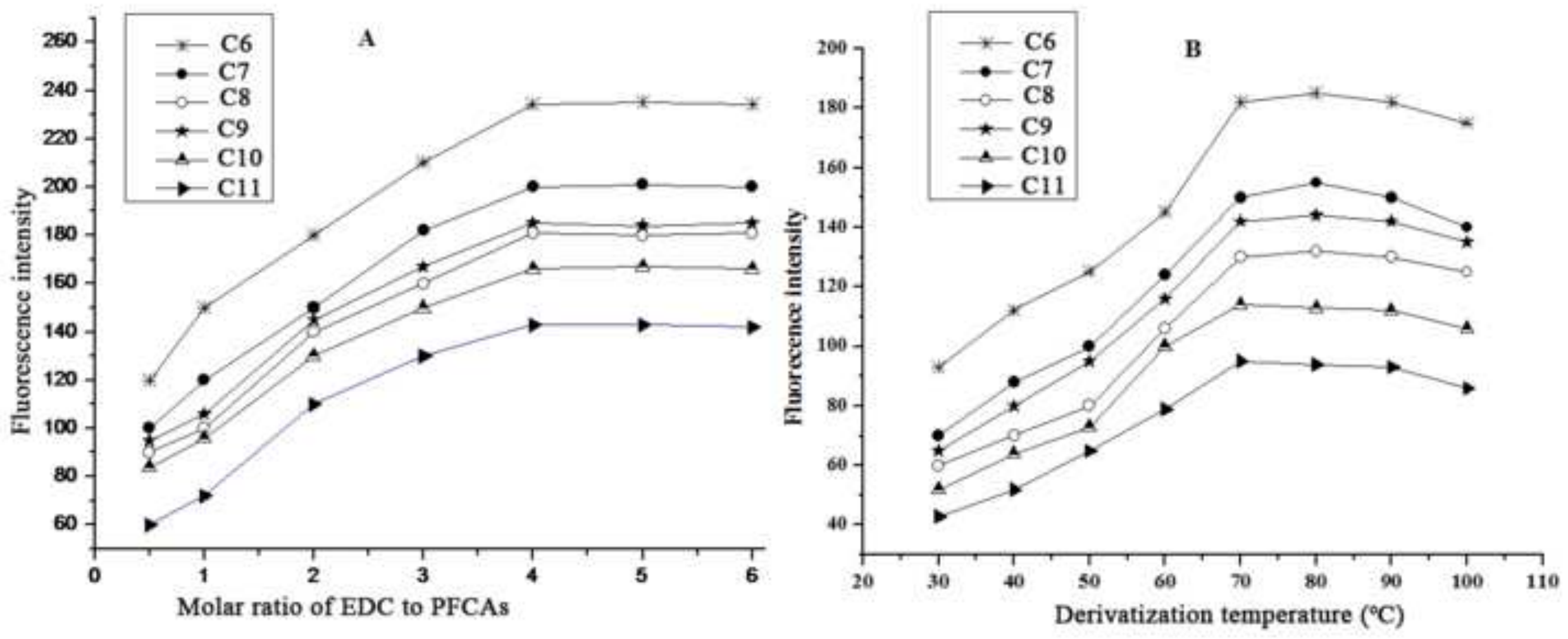


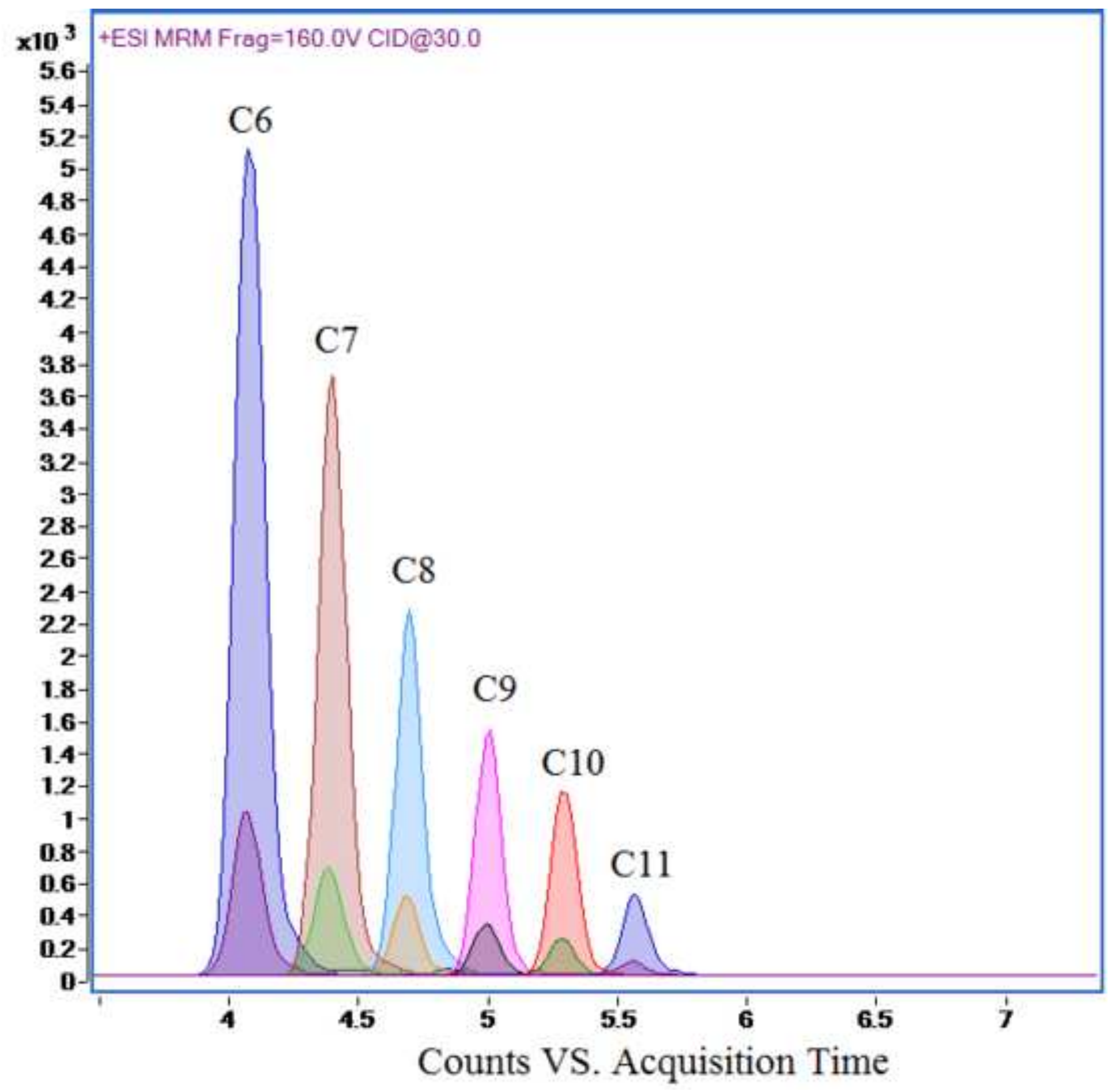


(A)

Light labeled sample

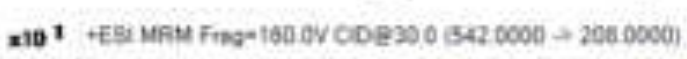

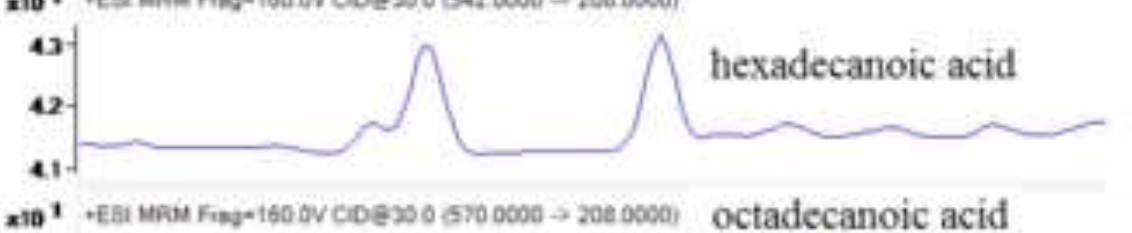

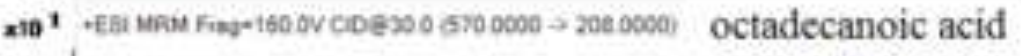

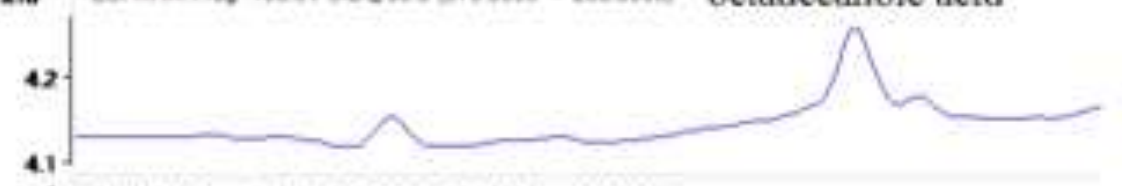

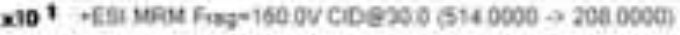

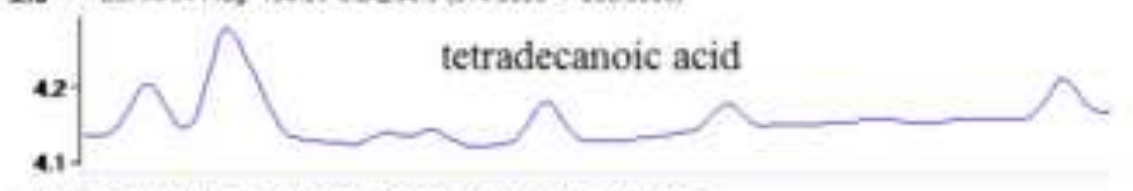

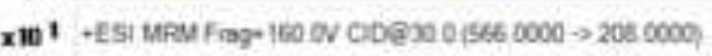

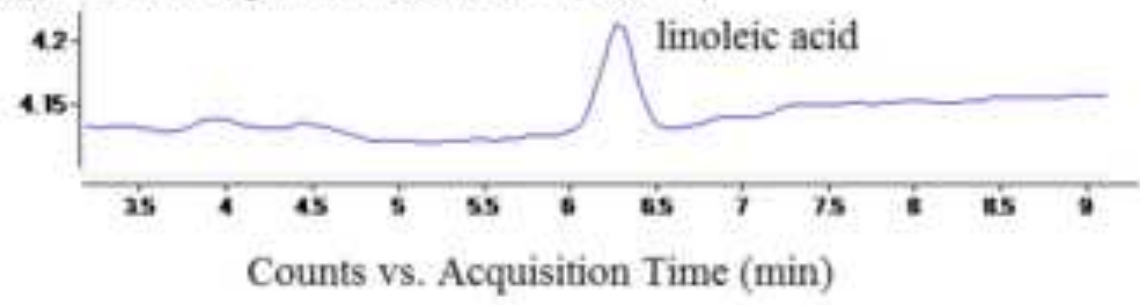

(B) heavy labeied standard

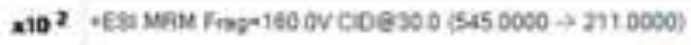

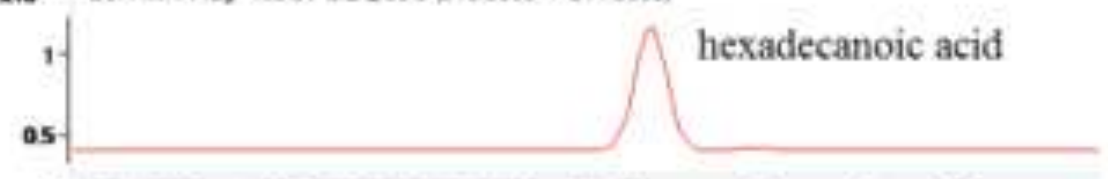

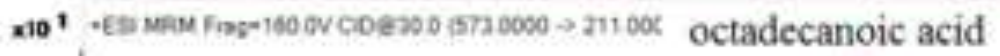

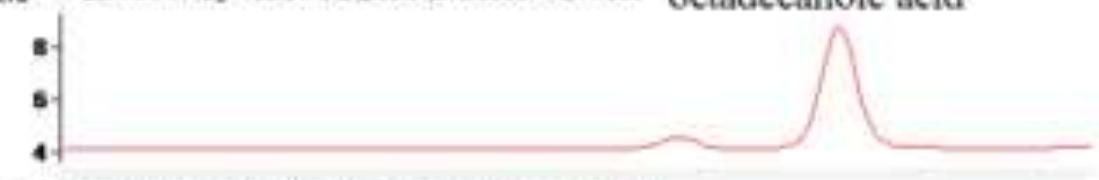

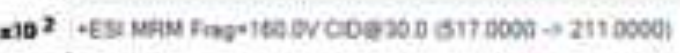

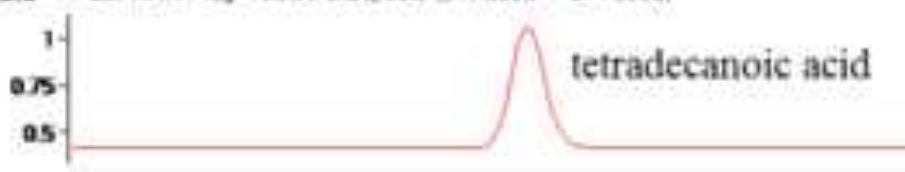

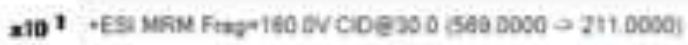

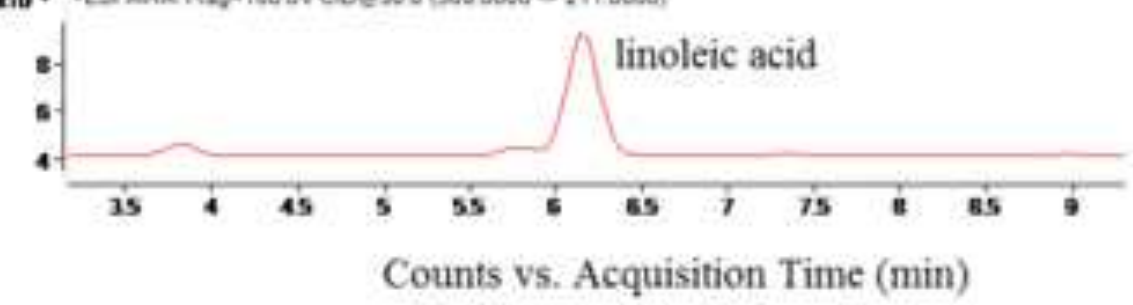




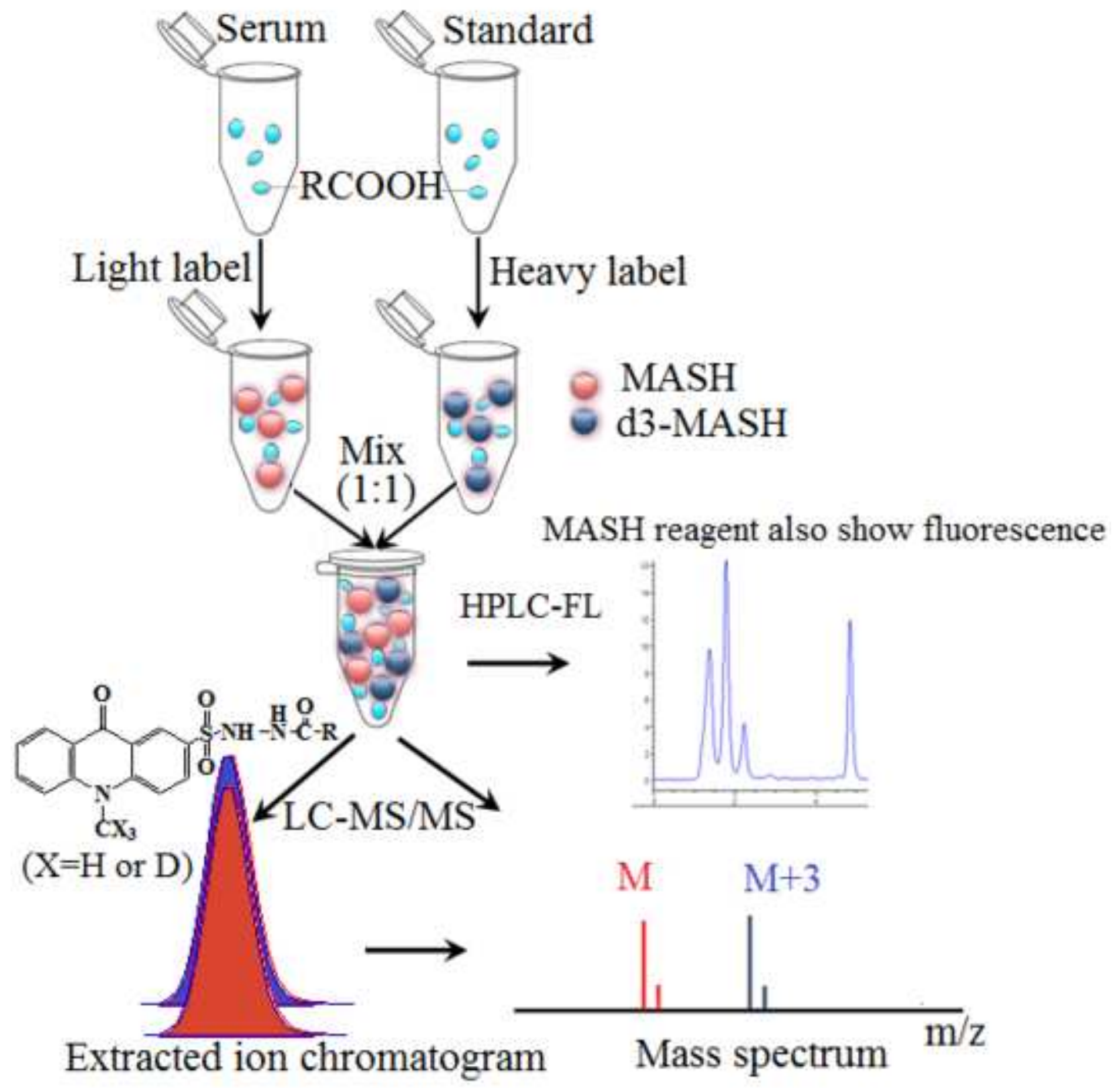

